\title{
EDITORIAL
}

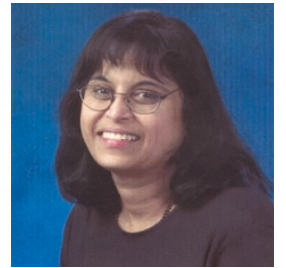

Sherin V. Devaskar, M.D.

\section{Pediatric Research - A look toward the future}

To the Pediatric Research Readership,

As I assume the role of Editor-in-Chief for the Pediatric Research Journal, I would like to take this opportunity to join Jean-Christophe Mercier, the European Chief Editor, in recognizing the significant contributions made by Alvin Zipursky and his colleagues during the last five years toward shaping the journal to its present form and standing. On the foundation that Alvin Zipursky, his colleagues, and their many predecessors have built, we would like to move forward by relying on the existent strengths and overcoming the major challenges we anticipate facing as a reflection of the present times.

We are in "the best of times and the worst of times" for pediatric research. Biologic sciences have seen a recent explosion in new knowledge that has expanded our horizons beyond prediction. Innovative technologies and an inter-disciplinary approach have allowed us to conquer problems facing science in a time-line considerably shorter than before, leading toward faster translation of observations from bench to bedside and back to the bench.

We are now in a postgenome era, in which we must untangle the reams of information gathered for the benefit of children and make practical sense of it. In addition, advancing technologies in areas of genomics, proteomics, transcriptomics, pharmacogenomics, epigenetics, metabolomics, nanosystems, and bioinformatics continue to enhance our ability to gather large amounts of novel information.

While we must continue to be great gatherers of information, it is essential that we stay in step as translators as well. Translation in the form of molecular physiology is critical as transgenic and gene knockout technologies are gaining a further sophistication (conditional, regulatable, knock-in approaches, and siRNA-transgenics) that did not exist before.

Clinical trials of sound experimental design based in developmental biology, physiology, and biochemistry will allow us to uncover the molecular, developmental, and physiologic causes behind various childhood disorders, ranging from genetic to environmental influences through the process of development. Novel bio-imaging technologies, biologic markers and tracers (e.g. stable isotopes), and genetic approaches have revolutionized clinical research as well. At the same time all our progress has raised a multitude of ethical, political, and social questions that we must prepare ourselves to address.

In this era of scientific abundance, we face a few difficult, but not insurmountable challenges. Today, when we have such a vast array of information and technology available to us, the funding needed to fuel our research endeavors has had diffi culty keeping pace. Equally of challenge, is the continuing need to strive toward more effective communication with and education of the general public regarding the practical meaning of the important scientific milestones achieved.

Our overall goal at the journal is to be a voice for scientists with a developmental focus in communicating their innovative observations, which form the basis for further research or lead to future interventions in developmental diseases. Toward meeting this end, our proximate goal is to focus on making the review process seamless. To accomplish this objective the first step has been to create a common editorial office for Pediatric Research, which will receive all manuscripts from around the world. The two editorial boards in North America and Europe will work in unison toward meeting our common objectives by using the overall existent expertise and beyond if necessary. The review process will strive toward taking the submission and review of manuscripts to a paperless, on-line system as soon as possible, which will decrease the turn-around time without compromising the quality or the even-handedness of the review process.

Our hope is that our efforts will continue to encourage scientists from all disciplines with a focus on developmental phenomena to submit their best work to Pediatric Research to further raise the standing of the journal. This will set the stage for progress as science evolves and keep the journal at the forefront of pediatric investigation.

Pediatric Research is overseen by the International Pediatric Research Foundation, but belongs to the membership of various academic societies that encompass different disciplines around the world with a commitment to pediatric research. We plan to steer the journal for the next five years to meet the expectations of the membership at large and make you proud of the accomplishments of the journal. We will continue to count 
on the assistance of the membership in this endeavor. In addition, we will reach out to those of you who are trainees and junior members who will shape the future of Pediatric Research to make the journal a home for your scientific contributions.

With your help, we expect to meet most of the challenges facing our journal. We are honored and appreciate the trust placed in us, and for the opportunity to represent Pediatric Research for the next term.

Sherin U. Devaskar, M.D. Editor-in-Chief Jean-Christophe Mercier, M.D. European Chief Editor 